\title{
Tubulointerstitial Fibrosis of Living Donor Kidneys Associates with Urinary Monocyte Chemoattractant Protein 1
}

\author{
Xiangling Wang ${ }^{a}$ John C. Lieske ${ }^{a, b}$ Mariam P. Alexander ${ }^{b}$ \\ Muthuvel Jayachandran ${ }^{c} \quad$ Aleksandar Denic $^{\mathrm{a}}$ Jerry Mathew ${ }^{\mathrm{a}}$ \\ Lilach O. Lerman $^{a}$ Walter K. Kremers ${ }^{d}$ Joseph J. Larson ${ }^{d}$ Andrew D. Rule ${ }^{a, d}$ \\ ${ }^{a}$ Division of Nephrology and Hypertension, Departments of b Laboratory Medicine and Pathology, 'Surgery, \\ Physiology and Biomedical Engineering, and ${ }^{\mathrm{d}}$ Health Sciences Research, Mayo Clinic, Rochester, Minn., USA
}

\section{Key Words}

Monocyte chemoattractant protein 1 . Collagen type IV .

Tubulointerstitial fibrosis · Implantation biopsies · Living kidney donors

\begin{abstract}
Background: Non-invasive biomarkers that detect occult pathology in patients with normal glomerular filtration rate (GFR) and normal urine albumin excretion may help identify patients at risk for chronic kidney diseases. Methods: Two promising biomarkers of interstitial fibrosis, urinary monocyte chemoattractant protein 1 (MCP-1) and collagen IV, were assayed among 634 living kidney donors from 2005 to 2011 , who had both a frozen pre-donation spot urine sample and a core needle biopsy of their donated kidney at transplantation ('time zero biopsy'). The association of urine MCP1 and collagen IV with kidney function (GFR and urine albumin excretion), kidney volume on computed tomographic imaging and histological findings was assessed. Results: The mean \pm SD age was $45 \pm 12$ years, 24 -hour urine albumin was $4 \pm 7 \mathrm{mg}$ and measured GFR (mGFR) was $102 \pm 18 \mathrm{ml} /$ $\mathrm{min} / 1.73 \mathrm{~m}^{2}$. The median (25th-75th percentile) urine level of MCP-1 was 146 (54-258) pg/ml and of collagen IV was 2.0 (1.0-3.5) $\mu \mathrm{g} / \mathrm{l}$. Higher urine MCP-1 associated with higher 24hour urine albumin excretion; higher urine collagen IV asso-
\end{abstract}

ciated with male gender. On kidney biopsy, any interstitial fibrosis was present in $22 \%$ and fibrosis $>5 \%$ in $4 \%$ of donors. The mean MCP- $1 / \mathrm{Cr}$ ratio was $1.49 \mathrm{pg} / \mathrm{mg}$ for $0 \%$ fibrosis, $1.80 \mathrm{pg} / \mathrm{mg}$ for $1-5 \%$ fibrosis, $2.33 \mathrm{pg} / \mathrm{mg}$ for $6-10 \%$ fibrosis and $4.33 \mathrm{pg} / \mathrm{mg}$ for $>10 \%$ fibrosis. After adjustment for age, sex, MGFR and 24-hour urine albumin, higher urine MCP-1 but not collagen IV associated with interstitial fibrosis and tubular atrophy. Conclusion: Urine MCP-1 may detect early tubulointerstitial fibrosis in adults with normal kidney function.

(c) 2016 S. Karger AG, Basel

\section{Introduction}

Renal tubulointerstitial fibrosis is a final common manifestation of chronic kidney disease (CKD) regardless of the etiology [1]. The degree and extent of tubulointerstitial fibrosis is a predictor of future renal function [2-4]. While moderate-severe interstitial fibrosis associates with CKD, mild interstitial fibrosis can be evident in patients with normal kidney function $[5,6]$. Early detection of renal fibrosis may be important for the timely initiation of intervention to prevent progression of kidney disease. Renal biopsy can be used to clinically assess renal fibrosis $[1,7]$, but is invasive and associated with a bleed-

\section{KARGER}

E-Mail karger@karger.com

www.karger.com/ajn
(C) 2016 S. Karger AG, Basel

0250-8095/16/0436-0454\$39.50/0
Andrew D. Rule

Division of Nephrology and Hypertension

Mayo Clinic 200 1st Street SW

Rochester, MN 55905 (USA)

E-Mail rule.andrew@mayo.edu 
ing risk that limits application in patients without a strong clinical indication. Thus, noninvasive markers that detect renal fibrosis in patients with otherwise normal kidney function are needed.

Studies have shown that inflammation contributes to the early pathogenesis of renal fibrosis $[3,8,9]$. Indeed, inflammatory chemokines, particularly monocyte chemoattractant protein 1 (MCP-1), detect interstitial inflammation and fibrosis in lupus nephritis [10]. Urine MCP-1 may have potential as a biomarker for diagnosis, prognosis and response to therapy in a variety of renal diseases including diabetic nephropathy and autosomal dominant polycystic kidney disease (ADPKD) [11-20]. Interestingly, urine MCP-1 can help detect early diabetic nephropathy lesions on biopsy prior to the proteinuric manifestations of the disease [21]. Type IV collagen is a major component of basement membranes [22] involved in the progression of renal fibrosis [1]. Studies have suggested urinary type IV collagen may be a predictor of $\mathrm{CKD}$ and its progression in diabetes [23-25]. However, the association of MCP- 1 and collagen IV levels with subclinical kidney remodeling remains unknown.

We hypothesized that urine MCP-1 and collagen IV levels may detect early tubulointerstitial fibrosis in adults with normal kidney function. We studied a sample of well-characterized living kidney donorscarefully screened for CKD risk factors and studied for kidney function. We tested the association of urine MCP-1 and collagen IV levels with kidney function, kidney volume on imaging and structural findings on kidney biopsy.

\section{Methods}

\section{Study Population}

This study was approved by the Institutional Review Board at the Mayo Clinic, Rochester Minnesota. Our study population consisted of living kidney donors evaluated at the Mayo Clinic (Rochester, Minn., USA) from 2005 to 2011 who had a pre-donation spot urine sample collected along with a core needle biopsy of their donated kidney during surgery. Each kidney donor underwent a renal CT angiography (CTA) scan and a standardized battery of tests as part of his or her pre-donation evaluation [26]. The kidney function tests included glomerular filtration rate (GFR) measured by iothalamate clearance and 24-hour urine albumin. Hypertension was defined by treatment with any antihypertensive therapy, systolic blood pressure $>140 \mathrm{~mm} \mathrm{Hg}$ or diastolic blood pressure $>90 \mathrm{~mm} \mathrm{Hg}$.

\section{Kidney Volume Measurement}

As described previously [27], kidney donors underwent a renal CTA scan as a part of pre-donation examination and the CT images from the angiogram phase were downloaded into a local workstation for processing. The kidney cortical and medullary vol- umes were estimated using image analysis software (ITK-SNAP version 2.2, University of Pennsylvania, Philadelphia, Pa., USA; www.itksnap.org) to semi-automatically segment the cortex and medulla from transverse images obtained during the angiogram (arterial) phase. The ratio of cortex volume to medulla volume was determined.

\section{Kidney Histology}

As described previously $[5,28]$, implantation renal biopsies were a routine part of the transplantation surgery intended to provide baseline histology for the recipient. An 18-gauge needle core biopsy of the cortex was taken with a biopsy gun $(1.7 \mathrm{~cm}$ specimen slot) at the time of implantation (intraoperative) after revascularization of the kidney in the recipient. The tissue specimen was fixed in formalin and embedded in paraffin. Nephron size on biopsy was subsequently characterized by mean glomerular (non-sclerotic) volume, cortex volume per glomerulus (inverse of glomerular density) and mean profile tubular area as previously described [28]. Nephrosclerosis on biopsy was also characterized by glomerulosclerosis, ischemic glomeruli, interstitial fibrosis and tubular atrophy and arteriosclerosis (intimal thickening or arteriolar hyalinosis). The number of non-sclerotic glomeruli, globally sclerotic glomeruli and ischemic glomeruli (identified by periglomerular fibrosis, capsule thickening and wrinkled tuft) were counted to determine percentage of globally sclerotic glomeruli and percentage of globally sclerotic and ischemic glomeruli. As previously described [6], a renal pathologist characterized the proportion of cortical area occupied by fibrosis $(0,1-5,6-10$, and $>10 \%)$ and number of distinct tubular atrophy/fibrosis foci on Mason's trichrome stained slides. Fibrosis was defined by interstitial expansion with increased collagen staining by trichrome. In this population, minimal or no inflammation was present in the fibrotic regions and any fibrosis was always focal rather than diffuse. Tubular atrophy/fibrosis foci were defined by the distinct regions within otherwise normal appearing parenchyma of thickened tubular basement membranes with surrounding fibrosis (regardless of size). The percentage of luminal stenosis was determined from the artery (if any) most orthogonal to its axis by the ratio of the intimal area to the intimal plus luminal area [6]. These measures of nephrosclerosis correlate with decreased kidney cortical to medullary volume ratio, increased kidney surface roughness and renal cysts [6]. Donors with a suboptimal biopsy section defined by compression artifact, $<2 \mathrm{~mm}^{2}$ of cortex or $<2$ glomeruli were excluded.

\section{Measurement of Urine MCP-1 and Collagen IV}

At the time of their pre-transplant evaluation, a spot urine sample from the donors was collected, centrifuged for $10 \mathrm{~min}$ at 2,000 $\mathrm{rpm}$ at $4^{\circ} \mathrm{C}$ and the supernatant was frozen at $-70^{\circ} \mathrm{C}$. Frozen aliquots for this study were subjected to one freeze-thaw cycle prior to this study. Urine MCP-1 was measured using the Quantikine Human CCL2/MCP1 ELISA kit (R\&D Systems, Minneapolis, Minn., USA) as previously described [29]. Urine collagen IV was measured using the type IV urinary collagen ELISA kit (Agrutus Medical, Daiichi Fine Chemical Co., Ltd., Japan). Urine creatinine was measured using the enzymatic creatinine kit (Roche Diagnostics, Indianapolis, Ind., USA). Duplicate measurements were performed for each urine specimen and those with a difference $>20 \%$ underwent repeat analysis. MCP- 1 and collagen IV concentrations were analyzed with and without normalization to urine creatinine. Both urine MCP-1 with and without normalization to urine cre- 
Table 1. Characteristics of living kidney donors $(n=634$ unless noted otherwise)

\begin{tabular}{|c|c|}
\hline Characteristics & Value \\
\hline \multicolumn{2}{|l|}{ Demographic } \\
\hline Mean age, years & $44.9 \pm 11.5$ \\
\hline Male & $272(43)$ \\
\hline White & $560(88)$ \\
\hline \multicolumn{2}{|l|}{ Kidney function and risk factors } \\
\hline $\mathrm{mGFR}^{*}, \mathrm{ml} / \mathrm{min} / 1.73 \mathrm{~m}^{2}$ & $102.2 \pm 17.6$ \\
\hline 24-Hour urine albumin**, mg & $4.3 \pm 7.3 ; 2.0(0.0-6.0)$ \\
\hline Hypertension & $94(15)$ \\
\hline BMI, $\mathrm{kg} / \mathrm{m}^{2}$ & $28 \pm 5$ \\
\hline \multicolumn{2}{|l|}{ Biopsy measures ${ }^{\#}$} \\
\hline \multicolumn{2}{|l|}{ Mean (non-sclerotic) glomerular } \\
\hline Cortex per glomerulus, $\mathrm{mm}^{3}$ & $0.07 \pm 0.05$ \\
\hline \multicolumn{2}{|l|}{ Mean profile tubular area } \\
\hline (including lumen), $\mathrm{mm}^{2}$ & $4,547.9 \pm 1,467.2$ \\
\hline \multicolumn{2}{|l|}{$\begin{array}{l}\text { Percentage of globally sclerotic } \\
\text { glomeruli }\end{array}$} \\
\hline 0 & $366(62)$ \\
\hline $1-5$ & $90(15)$ \\
\hline $6-10$ & $64(11)$ \\
\hline $11-15$ & $33(6)$ \\
\hline$>15$ & $41(7)$ \\
\hline \multicolumn{2}{|l|}{$\begin{array}{l}\text { Percentage of globally sclerotic/ } \\
\text { ischemic glomeruli }\end{array}$} \\
\hline 0 & $332(57)$ \\
\hline $1-5$ & $89(15)$ \\
\hline $6-10$ & $72(12)$ \\
\hline $11-15$ & $37(6)$ \\
\hline$>15$ & $54(9)$ \\
\hline \multicolumn{2}{|l|}{ Percentage of luminal stenosis } \\
\hline$<50$ & $406(76)$ \\
\hline $50-75$ & $110(21)$ \\
\hline$>75$ & $15(3)$ \\
\hline \multicolumn{2}{|l|}{ Percentage of cortical fibrosis } \\
\hline 0 & $463(79)$ \\
\hline $1-5$ & $104(18)$ \\
\hline $6-10$ & $16(3)$ \\
\hline$>10$ & $5(1)$ \\
\hline \multicolumn{2}{|l|}{$\begin{array}{l}\text { Number of fibrosis and atrophic } \\
\text { tubules foci }\end{array}$} \\
\hline 0 & $463(79)$ \\
\hline 1 & $83(14)$ \\
\hline $2+$ & $42(7)$ \\
\hline \multicolumn{2}{|l|}{ CT scan measures ${ }^{£}$} \\
\hline \multicolumn{2}{|l|}{ Total cortex volume } \\
\hline$(\text { left }+ \text { right })^{£ £}, \mathrm{ml}$ & $202 \pm 39$ \\
\hline \multicolumn{2}{|l|}{ Total medulla volume } \\
\hline (left + right $), \mathrm{ml}$ & $85 \pm 21$ \\
\hline Cortex/medulla volume & $2.5 \pm 0.6$ \\
\hline
\end{tabular}

Data are presented as the mean $\pm \mathrm{SD}$, median (25th-75th percentile) or $\mathrm{n}(\%)$. BMI $=$ Body mass index.

${ }^{*} \mathrm{n}=620 ;{ }^{* *} \mathrm{n}=602 ;{ }^{\#} \mathrm{n}=594 ;{ }^{£} \mathrm{n}=602 ;{ }^{£ £}$ cortical volume was directly segmented in 587 and estimated from total kidney volume in 15 donors. atinine have been presented in prior studies $[11,13-17,30]$. Normalization to urine creatinine addresses variation due to urine concentration, but urine creatinine also reflects muscle mass.

\section{Statistical Analysis}

All data are presented as the mean $\pm \mathrm{SD}$, median (25th-75th percentile) or n (\%). MCP-1, MCP-1/Cr, collagen IV and collagen IV/ $\mathrm{Cr}$ concentrations were natural $\log$ transformed for regression analysis. Linear regression models were used to determine the association of MCP-1, MCP-1/Cr, collagen IV and collagen IV/Cr with age, sex, kidney function, kidney volumes and kidney pathology (nephron size and nephrosclerosis measures). Regression slopes are described either per change of the original unit, per doubling or per SD of the original unit, according to which of these provided the best interpretation. Multivariate models with adjustment for age, sex, measured GFR (mGFR) and 24-hour urine albumin were performed.

\section{Results}

\section{Study Population}

Frozen urine was available for urinary MCP-1 and collagen IV measurement in 634 living kidney donors assessed between 2005 and 2011. Clinical, biopsy and CT characteristics of the 634 donors are shown in table 1 . The median (25th-75th percentile) urine level of MCP-1 was $146(54-258) \mathrm{pg} / \mathrm{ml}$ and of collagen IV was $2.0(1.0-3.5)$ $\mu \mathrm{g} / \mathrm{l}$.

\section{Association of Urine MCP-1 with Kidney Function, Volume and Biopsy Findings}

As shown in table 2, in unadjusted analysis, both urine $\mathrm{MCP}-1$ concentration and urine $\mathrm{MCP}-1 / \mathrm{Cr}$ ratio associated with higher urine albumin excretion, larger medullary volume and more tubulointerstitial fibrosis (either percentage of cortical fibrosis or number of tubular atrophy and interstitial fibrosis foci). Online supplemental figure 1 (for all online suppl. material, see www.karger.com/ doi/10.1159/000446851) shows MCP-1 concentration and $\mathrm{MCP}-1 / \mathrm{Cr}$ ratio increase with higher percentage of cortical fibrosis and with more tubular atrophy and interstitial fibrosis foci. The mean MCP-1/Cr ratio was $1.49 \mathrm{pg} /$ $\mathrm{mg}$ for $0 \%$ fibrosis, $1.80 \mathrm{pg} / \mathrm{mg}$ for $1-5 \%$ fibrosis, $2.33 \mathrm{pg} /$ $\mathrm{mg}$ for $6-10 \%$ fibrosis and $4.33 \mathrm{pg} / \mathrm{mg}$ for $>10 \%$ fibrosis. After adjustment for age, sex, mGFR and urine albumin excretion, both higher urine MCP-1 concentration and higher urine MCP-1/Cr ratio still associated with more tubulointerstitial fibrosis. The association of urine MCP-1 with urine albumin per doubling $(\beta=0.17, \mathrm{p}<0.0001)$ and the association of urine MCP-1/Cr with urine albumin per doubling ( $\beta=0.08, p<0.0001)$ remained statistically significant after adjusting for age, sex and mGFR. 
Table 2. Association of urine MCP-1 with kidney function, volume, and biopsy findings

\begin{tabular}{|c|c|c|c|c|c|c|c|c|}
\hline Characteristics & $\begin{array}{l}\text { unadjusted } \\
\beta \text { value }\end{array}$ & $\mathrm{p}$ value & $\begin{array}{l}\text { unadjusted } \\
\beta \text { value }\end{array}$ & $\mathrm{p}$ value & $\begin{array}{l}\text { adjusted } \\
\beta \text { value* }^{*}\end{array}$ & $\mathrm{p}$ value & $\begin{array}{l}\text { adjusted } \\
\beta \text { value* }\end{array}$ & $\mathrm{p}$ value \\
\hline Age, 10 years & -0.02 & 0.69 & 0.10 & $<0.0001$ & & & & \\
\hline Male & 0.51 & $<0.0001$ & -0.007 & 0.91 & & & & \\
\hline \multicolumn{9}{|l|}{ Kidney function and risk factors } \\
\hline Hypertension & 0.03 & 0.82 & 0.23 & 0.005 & -0.02 & 0.90 & 0.09 & 0.32 \\
\hline \multicolumn{9}{|l|}{ Kidney volume, SD } \\
\hline Cortical volume & 0.17 & 0.001 & 0.01 & 0.69 & 0.05 & 0.48 & 0.04 & 0.30 \\
\hline Medullary volume & 0.14 & 0.01 & 0.08 & 0.01 & 0.06 & 0.29 & 0.06 & 0.09 \\
\hline Cortex/medulla & 0.06 & 0.44 & -0.08 & 0.09 & 0.02 & 0.83 & -0.02 & 0.66 \\
\hline \multicolumn{9}{|l|}{ Nephron size, SD } \\
\hline Percentage of globally sclerotic glomeruli & 0.64 & 0.30 & 0.90 & 0.02 & 0.87 & 0.17 & 0.60 & 0.12 \\
\hline \multicolumn{9}{|l|}{ Percentage of globally sclerotic/ischemic } \\
\hline glomeruli & 0.81 & 0.15 & 0.95 & 0.005 & 0.95 & 0.11 & 0.63 & 0.08 \\
\hline Percentage of luminal stenosis & 0.10 & 0.68 & 0.18 & 0.22 & 0.11 & 0.66 & -0.02 & 0.88 \\
\hline Percentage of cortical fibrosis group & 0.24 & 0.01 & 0.23 & $<0.0001$ & 0.28 & 0.004 & 0.21 & 0.0005 \\
\hline \multicolumn{9}{|l|}{ Number of fibrosis and atrophic tubules } \\
\hline foci & 0.25 & 0.005 & 0.19 & 0.0004 & 0.28 & 0.002 & 0.16 & 0.005 \\
\hline
\end{tabular}

* Adjusted for age, sex, 24-hour urine albumin and mGFR.

\section{Association of Urine Collagen IV with Kidney}

Function, Volume and Biopsy Findings

As shown in online supplemental table 1, in unadjusted analysis, urine collagen IV concentration associated with younger age, male gender, higher urine albumin excretion, larger cortical volume, larger medullary volume, larger non sclerotic glomerular volume, larger cortex per glomerulus and larger mean profile tubular area. These associations were no longer evident (or were reversed) for urine collagen IV/Cr ratio. After adjustment for age, sex, mGFR and urine albumin excretion, higher urine collagen IV concentration associated with larger cortex per glomerulus and more globally sclerotic/ischemic glomeruli, while higher urine collagen IV/Cr associated with lower cortex/medulla ratio.

\section{Discussion}

We found that urine MCP-1, but not urine collagen IV level, was associated with tubulointerstitial fibrosis in a cohort of healthy living kidney donors. This finding was robust, as it was observed whether or not urine MCP-1 concentration was indexed to urine creatinine concentration and whether or not we adjusted for age, sex, GFR and urine albumin excretion. Kidney disease models implicate MCP-1 in the development of interstitial fibrosis via recruitment and activation of monocytes/macrophages [31-34]. Blockade of MCP-1/CCR2 signaling ameliorated renal fibrosis in a mouse unilateral ureteral obstruction nephropathy model associated with a decrease in type I collagen deposits and reduced TGF-beta expression [35].

Recently, multiplex-based cytokine profiling of urine demonstrated that MCP-1 performed better than a wide range of cytokines as a biomarker of SLE disease activity [30]. Studies implicate MCP-1 in the pathogenesis of tubulointerstitial damage in congenital obstructive nephropathy, where its urinary excretion may represent a powerful prognostic marker [36]. Studies in ADPKD revealed urinary excretion of MCP-1 was increased in the majority of adult patients [16] and was associated with total renal volume, independent of albuminuria [17]. In ANCA-associated vasculitis, urinary MCP-1 was useful to assess renal involvement and monitor the response to 
therapy [37]. In addition, studies in patients with type 2 diabetic nephropathy suggest that urinary MCP-1 excretion may be useful to evaluate the degree of renal fibrosis [38]. Furthermore, a recent study of normotensive and normoalbuminuric female patients with type 1 diabetes revealed that elevation of urinary MCP-1 excretion preceded clinical manifestations and was associated with the changes in kidney interstitial volume, but not glomerular lesions [21]. In the current study, we extend these observations to show that urine MCP-1 may constitute a biomarker of mild tubulointerstitial fibrosis among adults with normal kidney function.

Another notable finding was the association of urine MCP-1 with increased 24-hour urine albumin excretion even in this normal range for the healthy donors studied. Population studies have shown that modest increases in urine albumin excretion, well within the normal range of $<30 \mathrm{mg}$, are predictive of higher mortality and ESRD risk [39-41]. It is unclear if the source of the MCP-1 is interstitial versus glomerular, since increased glomerular leak of albumin or decreased tubular reabsorption could both result in increased urinary albumin excretion. Early studies implicated locally produced MCP-1 in the initiation and progression of tubulointerstitial damage [42, 43]. However, there has been an increasing body of evidence that MCP-1 also plays a role in the progression of glomerular lesions [31, 42, 44, 45]. Correlation of urine MCP-1 with urinary albumin or total protein excretion has been documented among patients with various kidney disorders $[13,46]$.

The other candidate urine biomarker in this study, urine collagen IV, associated with a lower cortex/medulla ratio even after adjustment for age, sex, mGFR and urine albumin. We have recently shown that a lower cortex/medulla ratio can be reflective of underlying tubulointerstitial fibrosis on biopsy [6], but we found no evi- dence that collagen IV associates with tubulointerstitial fibrosis on biopsy. One may hypothesize that urinary collagen IV may have more utility for detecting tubulointerstitial fibrosis in relatively more advanced kidney disease.

This study has certain limitations. The cohort consisted of healthy kidney donors, and thus per design all the pathology detected was relatively mild. The mild tubulointerstitial fibrosis in this population is unlikely to be of clinical significance without the onset of a separate progressive disease process. Furthermore, kidney needle core biopsies are a limited tissue specimen and this decreases the precision of the structural findings measured and the ability to assess the renal medulla. Nevertheless, our unique data set included a large number of biopsies, urine measures, kidney function measures and quantitative CT parameters in normal adults. Since biopsies are not available in normal individuals, use of biomarkers such as urine MCP-1 may allow the study of mild subclinical kidney pathology.

In conclusion, our study highlights MCP-1 as a useful marker to detect occult tubulointerstitial fibrosis. Urinary MCP-1 might allow further stratification of kidney function beyond the traditional measures of GFR and urinary albumin excretion.

\section{Acknowledgment}

This study was supported with funding from the National Institutes of Health, National Institute of Diabetes and Digestive and Kidney Diseases (R01 DK090358). The authors thank Michael Linnes for performing the ELISA analysis.

\section{Disclosure Statement}

All the authors declared no competing interests.

\section{References}

1 Genovese F, et al: The extracellular matrix in the kidney: a source of novel non-invasive biomarkers of kidney fibrosis? Fibrogenesis Tissue Repair 2014;7:4.

2 Declèves AE, Sharma K: Novel targets of antifibrotic and anti-inflammatory treatment in CKD. Nat Rev Nephrol 2014;10:257-267.

3 Liu Y: Renal fibrosis: new insights into the pathogenesis and therapeutics. Kidney Int 2006;69:213-217.

4 Wong MG, Pollock CA: Biomarkers in kidney fibrosis: are they useful? Kidney Int Suppl (2011) 2014;4:79-83.
5 Rule AD, et al: The association between age and nephrosclerosis on renal biopsy among healthy adults. Ann Intern Med 2010;152:561-567.

6 Denic A, Alexander MP, Kaushik V, Lerman LO, Lieske JC, Stegall MD, Larson JJ, Kremers WK, Vrtiska TJ, Chakkera HA, Poggio ED, Rule AD: Detection and clinical patterns of nephron hypertrophy and nephrosclerosis among apparently healthy adults. Am J Kidney Dis 2016;pii:S0272-6386(16)00020-2.

7 Hao W, Rovin BH, Friedman A: Mathematical model of renal interstitial fibrosis. Proc Natl Acad Sci U S A 2014;11:14193-14198.
8 Eddy AA: Molecular basis of renal fibrosis. Pediatr Nephrol 2000;15:290-301.

9 Hirschberg R: Wound healing in the kidney: complex interactions in renal interstitial fibrogenesis. J Am Soc Nephrol 2005;16:9-11.

10 Rovin $\mathrm{BH}$, et al: Urine chemokines as biomarkers of human systemic lupus erythematosus activity. J Am Soc Nephrol 2005; 16 : 467-473.

11 Wada T, et al: Up-regulation of monocyte chemoattractant protein-1 in tubulointerstitial lesions of human diabetic nephropathy. Kidney Int 2000;58:1492-1499. 
12 Kim MJ, Tam FW: Urinary monocyte chemoattractant protein-1 in renal disease. Clin Chim Acta 2011;412:2022-2030.

13 Banba N, et al: Possible relationship of monocyte chemoattractant protein-1 with diabetic nephropathy. Kidney Int 2000;58:684-690.

14 Morii T, et al: Association of monocyte chemoattractant protein-1 with renal tubular damage in diabetic nephropathy. J Diabetes Complications 2003;17:11-15.

$15 \mathrm{Wu} \mathrm{CC}$, et al: Aberrant cytokines/chemokines production correlate with proteinuria in patients with overt diabetic nephropathy. Clin Chim Acta 2010;411:700-704.

16 Zheng D, et al: Urinary excretion of monocyte chemoattractant protein-1 in autosomal dominant polycystic kidney disease. J Am Soc Nephrol 2003;14:2588-2595.

17 Meijer E, et al: Association of urinary biomarkers with disease severity in patients with autosomal dominant polycystic kidney disease: a cross-sectional analysis. Am J Kidney Dis 2010;56:883-895.

18 Tesch GH: MCP-1/CCL2: a new diagnostic marker and therapeutic target for progressive renal injury in diabetic nephropathy. Am J Physiol Renal Physiol 2008;294:F697-F701.

19 Camilla R, et al: Urinary monocyte chemotactic protein 1: marker of renal function decline in diabetic and nondiabetic proteinuric renal disease. J Nephrol 2011;24:60-67.

20 de Zeeuw D, et al: The effect of CCR2 inhibitor CCX140-B on residual albuminuria in patients with type 2 diabetes and nephropathy: a randomised trial. Lancet Diabetes Endocrinol 2015;3:687-696.

21 Fufaa GD, et al: Urinary monocyte chemoattractant protein-1 and hepcidin and early diabetic nephropathy lesions in type 1 diabetes mellitus. Nephrol Dial Transplant 2015;30: 599-606.

22 Zeisberg M, et al: Renal fibrosis: collagen composition and assembly regulates epithelial-mesenchymal transdifferentiation. Am J Pathol 2001;159:1313-1321.
23 Morita M, Hanai K, Uchigata Y: Urinary type IV collagen as a predictor for the incidence of microalbuminuria in young patients with type 1 diabetes. Diabet Med 2014;31:213-218.

24 Iijima T, et al: Follow-up study on urinary type IV collagen in patients with early stage diabetic nephropathy. J Clin Lab Anal 1998; 12:378-382.

25 Morita M, et al: Association of urinary type IV collagen with GFR decline in young patients with type 1 diabetes. Am J Kidney Dis 2011; 58:915-920.

26 Lorenz EC, et al: Prevalence of renal artery and kidney abnormalities by computed tomography among healthy adults. Clin J Am Soc Nephrol 2010;5:431-438.

27 Racusen LC, et al: The Banff 97 working classification of renal allograft pathology. Kidney Int 1999;55:713-723.

28 Elsherbiny HE, et al: Nephron hypertrophy and glomerulosclerosis and their association with kidney function and risk factors among living kidney donors. Clin J Am Soc Nephrol 2014;9:1892-1902.

29 Zhang X, et al: A composite urine biomarker reflects interstitial inflammation in lupus nephritis kidney biopsies. Kidney Int 2012;81: 401-406.

30 Barbado J, et al: MCP-1 in urine as biomarker of disease activity in systemic lupus erythematosus. Cytokine 2012;60:583-586.

31 Wada T, et al: Intervention of crescentic glomerulonephritis by antibodies to monocyte chemotactic and activating factor (MCAF/ MCP-1). FASEB J 1996;10:1418-1425.

32 Tang WW, et al: Chemokine gene expression in anti-glomerular basement membrane antibody glomerulonephritis. Am J Physiol 1995; 269(3 pt 2):F323-F330.

33 Natori Y, Sekiguchi M, Ou Z, Natori Y: Gene expression of CC chemokines in experimental crescentic glomerulonephritis (CGN). Clin Exp Immunol 1997;109:143-148.

34 Wada T, et al: MIP-1alpha and MCP-1 contribute to crescents and interstitial lesions in human crescentic glomerulonephritis. Kidney Int 1999;56:995-1003.

35 Wada T, et al: Gene therapy via blockade of monocyte chemoattractant protein-1 for renal fibrosis. J Am Soc Nephrol 2004;15:940948
36 Grandaliano G, et al: MCP-1 and EGF renal expression and urine excretion in human congenital obstructive nephropathy. Kidney Int 2000;58:182-192.

37 Tam FW, et al: Urinary monocyte chemoattractant protein-1 (MCP-1) is a marker of active renal vasculitis. Nephrol Dial Transplant 2004;19:2761-2768.

38 Tashiro K, et al: Urinary levels of monocyte chemoattractant protein-1 (MCP-1) and interleukin-8 (IL-8), and renal injuries in patients with type 2 diabetic nephropathy. J Clin Lab Anal 2002;16:1-4.

39 Brantsma $\mathrm{AH}$, et al: Urinary albumin excretion as a predictor of the development of hypertension in the general population. J Am Soc Nephrol 2006;17:331-335.

40 Gansevoort RT, de Jong PE: The case for using albuminuria in staging chronic kidney disease. J Am Soc Nephrol 2009;20:465-468.

41 Gutiérrez OM, et al: Racial differences in albuminuria, kidney function, and risk of stroke. Neurology 2012;79:1686-1692.

42 Viedt C, Orth SR: Monocyte chemoattractant protein-1 (MCP-1) in the kidney: does it more than simply attract monocytes? Nephrol Dial Transplant 2002;17:2043-2047.

43 Tesch GH, et al: Monocyte chemoattractant protein-1 promotes macrophage-mediated tubular injury, but not glomerular injury, in nephrotoxic serum nephritis. J Clin Invest 1999;103:73-80.

44 Panzer U, et al: Monocyte chemoattractant protein- 1 and osteopontin differentially regulate monocytes recruitment in experimental glomerulonephritis. Kidney Int 2001;59: 1762-1769.

45 Taal MW, et al: Proinflammatory gene expression and macrophage recruitment in the rat remnant kidney. Kidney Int 2000;58: 1664-1676

46 Rovin BH, Doe N, Tan LC: Monocyte chemoattractant protein-1 levels in patients with glomerular disease. Am J Kidney Dis 1996;27: 640-646.
Urinary MCP-1 Associates with

Tubulointerstitial Fibrosis
Am J Nephrol 2016;43:454-459

DOI: $10.1159 / 000446851$ 